\title{
Target DoA estimation in Passive Radar using non- uniform linear arrays and multiple frequency channels
}

\author{
Francesca Filippini, Tatiana Martelli, Fabiola Colone \\ DIET Department, Sapienza University of Rome \\ Via Eudossiana 18, 00184 Rome (Italy) \\ \{francesca.filippini, tatiana.martelli, fabiola.colone\}@uniroma1.it
}

\author{
Roberta Cardinali \\ Leonardo Company \\ Via Tiburtina Km 12.400, 00131 Rome (Italy) \\ roberta.cardinali@leonardocompany.com
}

\begin{abstract}
In this paper we present a robust approach for target direction of arrival (DoA) estimation in passive radar that jointly exploits spatial and frequency diversity. Specifically we refer to a DVB-T based passive radar receiver equipped with a linear array of few antenna elements non-uniformly spaced in the horizontal dimension, able to collect multiple DVB-T channels simultaneously. We resort to a maximum likelihood (ML) approach to jointly exploit the target echoes collected across the antenna elements at multiple carrier frequencies. Along with an expected improvement in terms of DoA estimation accuracy, we show that the available spatial and frequency diversity can be fruitfully exploited to extend the unambiguous angular sector useful for DoA estimation, which represent an invaluable tool in many applications. To this purpose, a performance analysis is reported against experimental data collected by a multi-channel DVB-T based passive radar developed by Leonardo S.p.A.
\end{abstract}

Keywords-passive radar, direction of arrival (DoA), DVB-T signals, multi-frequency, non uniform linear array.

\section{INTRODUCTION}

Passive coherent location (PCL) technology has been extensively studied for the last decades due to the several advantages it offers over active radars [1]. Despite the limitations yet being investigated, the relevant technical literature continuously reports new attempts to identify effective signal processing techniques and operative strategies to make these systems work in different scenarios.

Due to the weak target echoes that PCL systems typically receive, usually embedded in the background of strong direct signal, multipath and noise, accurate target localization may represent a challenging task. This is also exacerbated by the low carrier frequencies that are exploited by the transmissions of opportunity usually employed for long range surveillance applications. Target localization might be improved by exploiting a passive radar tracking network; however the data association stage might be hobbled by the localization performance of the single sensor within the network.

When a single PCL sensor is deployed, a 2D target localization is usually accomplished based on measurements of bistatic range and direction of arrival (DoA). With particular reference to a digital video broadcasting terrestrial (DVB-T) based PCL system, the target range estimation is indeed facilitated by the larger signal bandwidth with respect to other waveforms of opportunity usually employed for wide area coverage applications, e.g. FM radio, DAB, etc. However, target DoA estimation is characterized by low accuracies even operating at UHF band. This especially applies to PCL systems employing a limited number of receiving channels connected to few antenna elements in order to limit the system complexity.
In fact, in such systems, the requirements of accurate DoA estimation and wide unambiguous angular sector sets competing constraints on the array equivalent length. Basically, when operating with a uniform linear array (ULA), the need to guarantee a wide unambiguous angular sector sets an upper limit on the inter-element spacing. However, if a small number of elements is employed, this would result in an array of reduced size and, in turn, in a poor estimation accuracy. In contrast, the presence of ambiguities in DoA estimation has to be accepted in order to reduce the estimation errors for unambiguous targets.

A typical design solution is to set the unambiguous angular sector to be comparable to the beamwidth of the employed antenna elements so that targets that are likely to be detected can be unambiguously localized. However, in applications involving simultaneous short and long-range coverage, for instance in coastal surveillance, there is a high probability to detect close targets through the sidelobes of the antenna elements.

In this paper, we investigate the possibility to overcome the limitations above by resorting to a non-uniform arrangement of the antenna elements and jointly exploiting the signals received at different carrier frequencies, which is a typical condition offered when exploiting broadcast transmissions as sources of opportunity.

The exploitation of sparse arrays of sensors has been investigated in many fields, e.g. radar, sonar, communications, etc., aiming at relaxing the constraint on the spatial sampling [2]. In such systems, the concept of ambiguous estimate takes a stochastic perspective and it is often referred as outlier. The probability of outliers depends on the target signal-to-noise ratio (SNR) and on the array layout [3].

The possibility of jointly exploiting multiple signals emitted by the same broadcast illuminator of opportunity (IO) at different carriers has been investigated in [5]-[10]. It has been shown that frequency diversity can be effectively exploited in order to improve the target detection capability and the reliability of the PCL system [5]-[6]. Suitable strategies have also been devised aiming at achieving an improved range resolution [7]-[9]. Among the different broadcast IOs, DVB-T is especially attractive for multifrequency (MF) operations, due to the wide frequency band allocated to the DVB-T service in the UHF band (from 470 $\mathrm{MHz}$ to $862 \mathrm{MHz}$ ). In fact, along with the benefits that the signal modulation offers for PCL applications, e.g. ambiguity function with a thumb-tack shape and constant bandwidth, also a large frequency diversity can be experienced. Based on the several benefits offered by MF approaches, we extend them to target DoA estimation. 
A first attempt in this direction has been proposed in [10] for FM radio based PCL, aiming at improving the angular localization accuracy and making the DoA estimation performance robust with respect to the time-varying characteristics of the waveforms.

In this work, the MF operation is considered in conjunction with a non-uniform linear array (NULA) layout in order to relax the trade-off between DoA estimation accuracy and unambiguous angular sector. Specifically by resorting to a maximum likelihood (ML) approach that incoherently integrates the target echoes at multiple carrier frequencies, we benefit from the increased equivalent target SNR that provides enhanced estimation accuracy for unambiguous targets. However, along with this expected advantage, the frequency diversity allows to build up an improved ML criterion function showing reduced sidelobes level. Therefore, the probability of outliers in target DoA estimation is lowered even operating with few and largely spaced antenna elements. As is well known, other strategies can be used to fruitfully exploit the baseline diversity, such as the MVDR algorithm, the MUSIC algorithm etc., [4]. In this work, we used the classical ML approach in order to investigate the benefits yield by the joint exploitation of frequency diversity and NULA.

The application against experimental data sets provided by Leonardo S.p.A. allows to demonstrate that the MF operation yields a significant reduction of the outliers even employing just two antenna elements on receive, mounted at a distance higher than the wavelength. Moreover, by considering a slightly increased array size of three elements and jointly exploiting frequency diversity and spatial diversity, the probability of outliers is further reduced and target localization accuracy is largely enhanced.

The paper is organized as follows. In Section II, the proposed DoA estimation approach is described and its theoretical benefits are presented in Section III. An experimental validation is reported in Section IV, where the data set is described and some results are shown. Eventually, some concluding remarks are drawn in Section V.

\section{Multi-FreQuenCy ML DoA Estimation}

Let us consider the availability of one reference antenna, steered toward the exploited IO and $K$ surveillance antennas, steered toward the area to be monitored and able to simultaneously collect signals transmitted at $N$ different carrier frequencies by the same emitter. First, each of the $K N$ collected signals separately undergoes the main processing stages of a conventional multi-channel DVB-T based PCL system, as sketched in Fig. 1.

First of all, in order to remove the direct signal, clutter and multi-path contributions, a disturbance cancellation stage is performed. Then, the output of the first stage and the corresponding reference signal are used in order to evaluate the bistatic range-velocity map, where target contributions possibly appear as delayed and Doppler shifted peaks.

A non-coherent integration (NCI) across the $N$ DVB-T channels is then applied, followed by a Multi-Frequency CellAverage Constant False Alarm Rate (CA-CFAR) detection scheme [5]. Then, an $M$-out-of- $K$ criterion is used to integrate the detection results over the $K$ surveillance channels; namely, a detection is declared at a range-velocity location that exceeds the threshold at $M$ surveillance channels out of the $K$

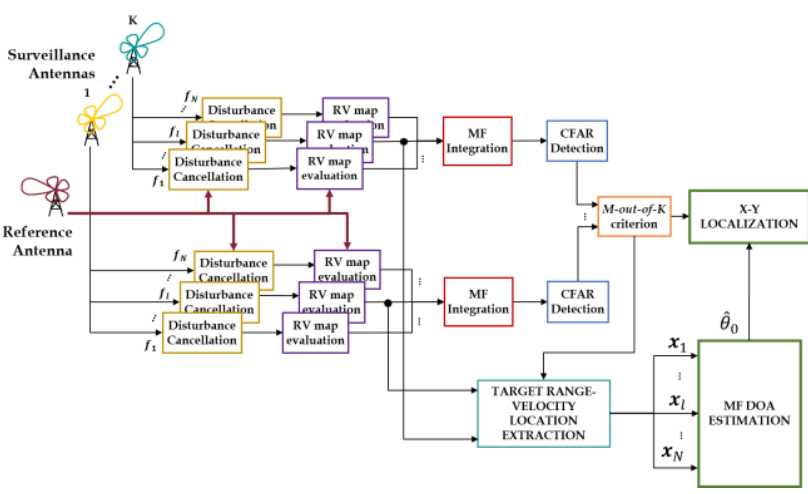

Fig. 1 Processing scheme for a PCL system exploiting multiple DVB-T channels

available. See [6] and the reference therein for further details on the signal model and the processing stages required for $\mathrm{MF}$ target detection. Once the target has been detected on the range-velocity plane, the DoA of the corresponding echo has to be estimated with the purpose of localizing it in the X-Y plane. For the $l$-th frequency channel $(l=1, \ldots, N)$, we consider the $K$ range-velocity maps relative to the $K$ surveillance antennas, and we assume to collect in a vector $\mathbf{x}_{l}$ the complex values extracted at the range-velocity location where the target has been detected. The vector $\mathbf{x}_{l}$ includes both target contribution and noise and it is written as:

$$
\mathbf{x}_{l}=A_{l} \mathbf{s}_{l}\left(u_{0}\right)+\mathbf{n}_{l} \quad(l=1, \ldots, N)
$$

where

- $A_{l}$ is the deterministic (but unknown) complex target amplitude at the $l$-th frequency channel. We assume the target amplitude values (both modulus and phase) are different at the different frequency channels. Notice that this hypothesis excludes the possibility of coherently integrating the target echoes received across DVB-T frequency channels.

- $\mathbf{s}_{l}\left(u_{0}\right)$ is the target steering vector and models the array response to a unit waveform from the target DoA $u_{0}$. For a linear array composed by $K$ surveillance antennas at positions $d_{k}(k=0, \ldots, K-1)$ measured with respect to the phase reference point, $\mathbf{s}_{l}\left(u_{0}\right)$ is:

$$
\mathbf{s}_{l}\left(u_{0}\right)=\left[e^{\left(j \frac{2 \pi}{\lambda_{l}} d_{0} u_{0}\right)} \quad \ldots \quad e^{\left(j \frac{2 \pi}{\lambda_{l}} d_{K-1} u_{0}\right)}\right]
$$

where $u_{0}=\sin \left(\theta_{0}\right)$, with $\theta_{0}$ equal to the target angle of arrival, measured relative to the array boresight and $\lambda_{l}$ is the $l$-th wavelength.

- $\mathbf{n}_{l}$ contains the noise samples collected at the $K$ surveillance channels. Under the assumption of an effective cancellation of the disturbance contributions, we can assume $\mathbf{n}_{l}$ to be a $K$-dimensional complex Gaussian random variable with zero mean and covariance matrix $\sigma_{n, l}^{2} \mathbf{I}_{K}$.

We furthermore assume that the noise samples at different frequency channels are statistically independent. The joint probability density function (pdf) of the signals collected at the $N$ frequency channels under hypothesis $H_{\gamma}\left(H_{1}\right.$ :target + noise; $H_{0}$ : noise only) can be written as:

$$
\begin{gathered}
p_{\mathbf{x}_{0}, ., \mathbf{x}_{N-1}}\left(\mathbf{x}_{0}, \ldots, \mathbf{x}_{N-1} \mid H_{\gamma}\right)= \\
\left(\pi \sigma_{n, l}^{2}\right)^{-K N} \exp \left\{-\sigma_{n, l}^{-2} \sum_{l=0}^{N-1}\left|\mathbf{x}_{l}-\gamma A_{l} \mathbf{s}_{l}\left(u_{0}\right)\right|\right\}
\end{gathered}
$$


Therefore, the ML estimate of the target DoA is found by jointly maximizing the above pdf with respect to $A_{l}(l=$ $1, \ldots, N)$ and $u_{0}$, yielding

$$
\hat{u}_{0}=\max _{u}(V(u))
$$

where $V(u)$ is the ML criterion function and is given by

$$
V(u)=\sum_{l=0}^{N-1} \frac{1}{\sigma_{n, l}^{2}}\left|\mathbf{s}_{l}{ }^{\mathrm{H}}(u) \mathbf{x}_{l}\right|^{2}
$$

\section{ILLUSTRATION OF THE BENEFITS OF THE PROPOSED DOA ESTIMATION APPROACH}

The target DoA estimation scheme in the previous Section is well suited for any surveillance array layout and for any DVB-T channels selection. However, such parameters highly affect the achievable performance in terms of both estimation accuracy and unambiguous angular sector width.

In this Section, we consider different configurations in order to investigate the benefits of the proposed approach. The case studies considered have been set according to the parameters adopted in the experimental tests reported in Section IV. Nevertheless, it is worth noticing that both the spacing between the antennas and the DVB-T channels selection could be optimized to improve the estimation accuracy or to reduce the probability of outliers [3].

\section{a) Two surveillance antennas and a single DVB-T channel}

First, we consider two receiving antennas $(K=2)$ and the exploitation of a single DVB-T channel $(N=1)$. As is well known, in this case, the ML estimator in (4) corresponds to a simple interferometric approach that exploits the phase difference of arrival $\phi$ between the two receiving antennas at relative distance $d=d_{1}-d_{0}$ to estimate the target DoA by inverting the following relation:

$$
\phi=[2 \pi d / \lambda] \sin \left(\theta_{0}\right)
$$

Notice that, in this simple case, the likelihood function in (5) has a periodic behavior that depends on the ratio $d / \lambda$. This is sketched in dashed blue curve in Fig. 2 for the case of a target at $\theta_{0}=30^{\circ}$ in a noise-free scenario. The curve has been obtained by considering the exploitation of the DVB-T

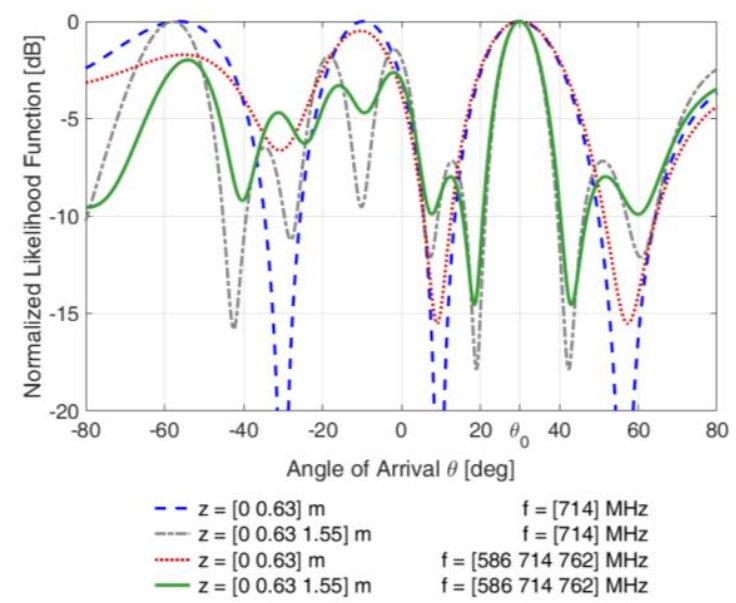

Fig. 2 Normalized likelihood function for a target with $\theta_{0}=30^{\circ}$ in a noise-free scenario, as a function of the angle of arrival $\theta$, using

- a single DVB-T channel with two (dashed blue) or three (dashdot gray) surveillance antennas

- three DVB-T channels with two (dotted red) or three (green) surveillance antennas. channel at $f_{c}=714 \mathrm{MHz}$ and spacing $d=0.63 \mathrm{~m}=1.5 \lambda$ between the antennas. As is apparent, the main peak appears at the true angle of arrival $\theta_{0}$.

However, as is expected, two additional peaks, i.e. grating lobes, are visible at $\theta$ such that $\sin (\theta)=\sin \left(\theta_{0}\right)+k \lambda / d$, where $k$ is an integer. Therefore, in order to avoid ambiguities, the maximization of (5) has to be limited within the angular sector $\Delta \theta=2 \sin ^{-1}(\lambda / 2 d)$ that in the considered case is equal to $38.95^{\circ}$.

\section{b) Three surveillance antennas and a single DVB-T channel}

Let us now consider the availability of three surveillance antennas $(K=3)$ and still a single DVB-T channel. The main advantage when exploiting more antenna elements is the achievement of a narrower main lobe, thanks to the increase of the equivalent array size, which results in a better accuracy in the DoA estimation. However, it is worth recalling that if the sensors remain equally spaced, grating lobes still appear at the same positions, namely the unambiguous angular sector is still narrow.

Therefore, in order to increase at the same time both the DoA estimation accuracy and the unambiguous angular sector, a NULA can be used. The corresponding likelihood function is illustrated in Fig. 2 in dash-dot gray, for a target with angle of arrival $\theta_{0}$ and elements positions $\boldsymbol{z}=$ $\left[\begin{array}{lll}d_{0} & d_{1} & d_{2}\end{array}\right]=\left[\begin{array}{lll}0 & 0.63 & 1.55\end{array}\right] \mathrm{m}$. By observing Fig. 2, both benefits are evident with respect to the case of $K=2$. In fact, (i) a narrow main lobe is obtained, which means that a better estimation accuracy is offered; (ii) a wider unambiguous angular sector is gained. In fact, the first grating lobe now appears at $\theta=-59^{\circ}$, namely at a distance of approx. $89^{\circ}$ from the main lobe. Therefore, the maximum of (5) can be searched for in a wider angular sector.

Nevertheless, some additional sidelobes appear close to the main lobe, with a level just $1.4 \mathrm{~dB}$ below the main peak. Although, strictly speaking, they are not grating lobes, these high peaks could yield an incorrect DoA estimation, depending on the noise power level, i.e. there is a non-zero probability to obtain ambiguous results. Moreover this probability increases as the target SNR decreases.

These estimation errors, often referred as outliers, have been studied in [3] where a theoretical performance prediction is provided for a ML DoA estimator operating at low SNR values, i.e. in the so called threshold region. The results in [3] can be fruitfully exploited to optimize the sensors spacing, in order to guarantee a desired probability of outliers. However, when few antenna elements are employed, the optimization would certainly lead to a reduction of the array length or, alternatively, to an increase of the number of elements within the array.

\section{c) Multiple DVB-T channels exploitation}

Let us now assume to be able to collect multiple frequency channels simultaneously. To this purpose, with reference to the real data analyzed in Section IV, three DVB-T channels within the UHF band have been considered, at carrier frequencies $\{586,714,762\} \mathrm{MHz}$.

First, we consider the case of two surveillance antennas with spacing $\mathrm{d}=0.63 \mathrm{~m}$. Notice that the spacing between the two receiving sensors is higher than all the considered 
wavelengths. In a noise-free scenario and assuming comparable target power levels at the different frequency channels, the likelihood function derived from their joint exploitation for target DoA estimation, is sketched in dotted red in Fig. 2. As is apparent, no grating lobes appear in the considered angular region. Indeed, each of the selected DVB$\mathrm{T}$ channels would offer different ambiguous DoAs, i.e. different positions of the grating lobes in the resulting SF likelihood function. Therefore, if the frequencies are properly chosen, their integration benefits of such diversity and allows mitigating this effect.

However, it is worth noticing that, for the considered selection, a high sidelobe is visible at approx. $0.4 \mathrm{~dB}$ from the main peak, which could result in outliers as the SNR decreases. Moreover, since a non-coherent integration is performed across the available frequency channels, a possible improvement in term of DoA estimation accuracy has to be attributed mainly to the equivalent SNR increase rather than to an improvement of the ML criterion function. In other words, the width of the likelihood mainlobe will certainly degrade with respect to that offered by the highest carrier frequency.

It is worth noticing that a better selection of the DVB-T channels can be performed to control the above effects. Moreover, the DVB-T band potentially offers a wide diversity. However, operating with signals of opportunity, one cannot totally control the frequencies to be integrated, but can only choose a subset of frequencies from those emitted by the selected IO.

Hence, along with the different frequencies, a crucial degree of freedom to lower the probability of outliers lies in the array layout. For this reason, we jointly consider the availability of three surveillance antennas, located at positions $\boldsymbol{Z}=\left[\begin{array}{lll}0 & 0.63 & 1.55\end{array}\right] \mathrm{m}$ and three DVB-T channels. The corresponding likelihood function is illustrated in Fig. 2 in green and the benefits are evident. In fact, along with the absence of grating lobes in the considered angular region, the sidelobes are kept lower than $2.5 \mathrm{~dB}$ with respect to the main peak, thus reducing the probability of incorrect DoA estimation. Moreover, the narrow main lobe guarantees an improved DoA estimation accuracy with respect to the use of a pair of surveillance antennas.

\section{EXPERIMENTAL VALIDATION}

In this Section, we show the results from the application of MF approaches against data collected by a DVB-T based PCL system in an aerial surveillance scenario. Experimental data, provided by Leonardo S.p.A., have been processed in order to show the benefits of the proposed DoA estimation technique.

\section{A. Acquisition campaign}

The acquisition campaign has been carried out on May 2016 at the Leonardo S.p.A. premises in Rome (see Fig. 3). The reference antenna was steered toward the exploited DVBT IO located in Monte Cavo Vetta, approx. $22.5 \mathrm{~km}$ from the receiver site. Three Yagi-Uda surveillance antennas were employed, pointed at $258^{\circ}$ clockwise from North and aligned in the horizontal plane; each antenna element has a main beam width of approx. $36^{\circ}$. As is apparent by looking at the geometry in Fig. 3, the main beam of the surveillance

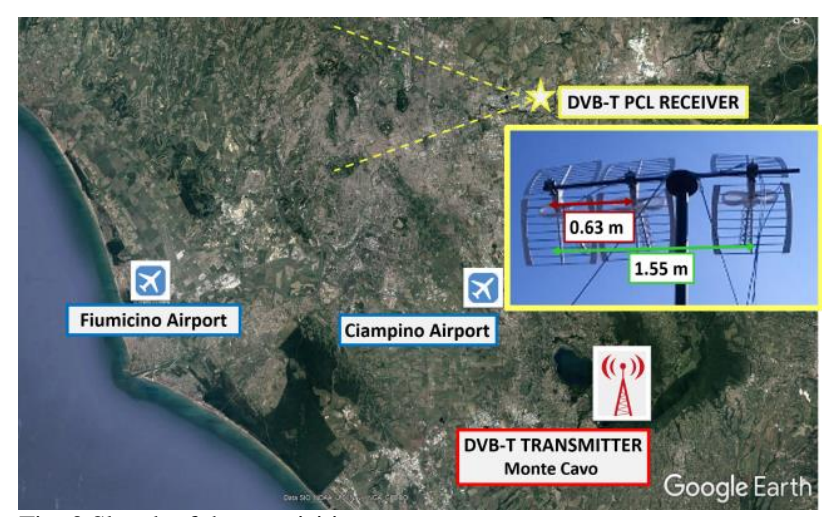

Fig. 3 Sketch of the acquisition geometry.

antennas include a section of the civilian air traffic departing or arriving at Fiumicino and Ciampino airports.

Two different configurations of surveillance antennas are considered, namely two receiving sensors with positions $\boldsymbol{Z}=$ $\left[\begin{array}{ll}0 & 0.63\end{array}\right] \mathrm{m}$ and three antennas with positions $\boldsymbol{z}=$ $\left[\begin{array}{lll}0 & 0.63 & 1.55\end{array}\right] \mathrm{m}$ (as sketched in Fig. 3). Several data sets have been collected, each composed by sequential data files of temporal duration equal to $0.45 \mathrm{~s}$, spaced by approx. $4 \mathrm{~s}$.

Different DVB-T channels within the UHF band, namely $\left\{f_{1}, f_{2}, f_{3}\right\}=\{586,714,762\} \mathrm{MHz}$, have been collected simultaneously. Live Air Traffic Control (ATC) registrations of the aircrafts present in the same area have been also collected. The availability of air-truth for the noncooperative targets allowed us to carry out a quantitative analysis to evaluate the performance improvement. A calibration of the system has been performed based on the position of the IO and on the air traffic of opportunity, over some hours of acquisitions.

\section{B. Experimental Results}

First, the use of the single DVB-T channel is considered for the case of two or three surveillance antennas. All the available data files of each carrier frequency have been separately processed, according to the basic DVB-T PCL processing scheme described in Section II. In particular, the ECA [11] is applied with 1000 taps (i.e. $33 \mathrm{~km} @ f_{S}=$ 64/7MHz).

A Coherent Integration Processing (CPI) of $0.3 \mathrm{~s}$ is used to evaluate the range-velocity maps and target detection is performed by resorting to a CA-CFAR with a probability of false alarm equal to $P_{F A}=10^{-3}$ and $P_{F A}=10^{-2}$ on each surveillance channel when two and three antennas are employed, respectively. Then, after a 2 -out-of-2 or a 3-out-of3 detection criterion across the surveillance channels, a nominal $P_{F A}=10^{-6}$ is obtained on the final range-velocity plane in all cases.

As an example, in this paper, we focus on a single target track, potentially observed at 34 consecutive data files (which correspond to approx.136 s). Fig.4(a) reports the localization results over the X-Y plane for the considered target, while in Fig.4(b) the same plots are reported as a function of the acquisition time. In this case, the single DVB-T channel at $f_{2}=714 \mathrm{MHz}$ is exploited and the signals from two surveillance antennas are employed. The PCL plots are shown in red dots, the air-truth is reported in black for comparison and, in Fig.4(a), the main beam and the iso-bistatic range ellipses are sketched in dashed dark blue and light blue dots, respectively. 


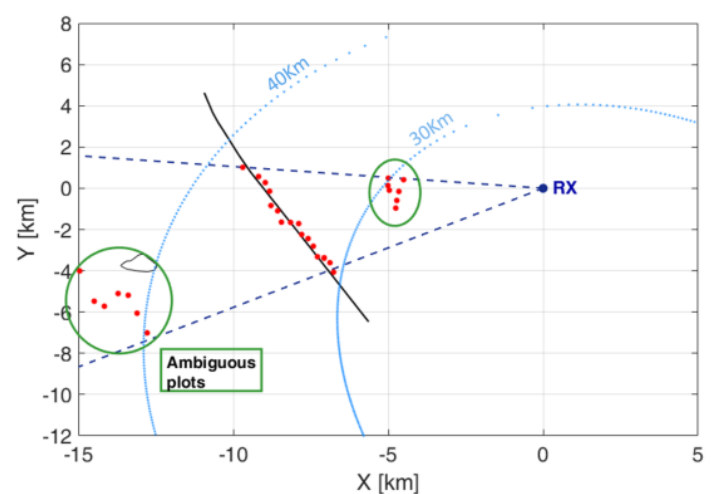

(a)

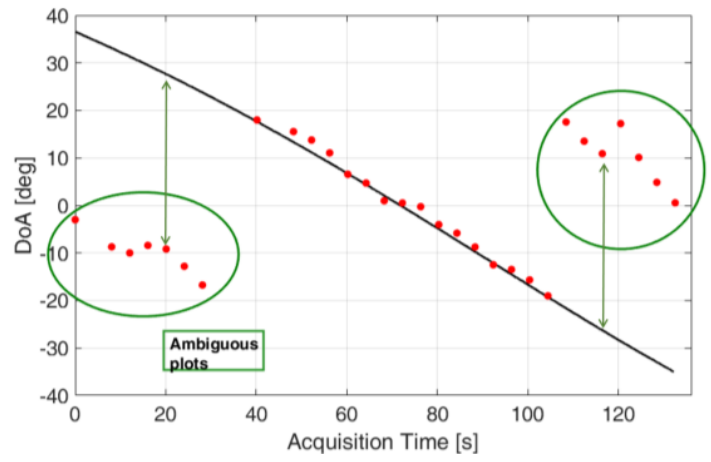

(b)

Fig.4. Localization results for 34 consecutive data files using two surveillance antennas and exploiting the best single DVB-T channel

$\left[f_{2}\right]$ : (a) X-Y plane, (b) estimated DoA vs acquisition time.

Specifically, $f_{2}$ represents the best performing DVB-T channel in terms of target detection capability (see Table I). Notice that for the considered DVB-T channel, when two antennas are employed, the target DoA is estimated unambiguously only in a narrow angular sector ( see Section II ). Therefore, the PCL plots located outside this sector are mis-localized, as highlighted by the green circles in Fig.4 (a) and Fig.4 (b). As is apparent by observing Fig.4, the localization errors are exclusively due to ambiguous angle measurements since the ambiguous plots lie on the correct iso - bistatic range ellipses but their estimated DoA show a distance of approx. $39^{\circ}$ from the real one, as expected for the ambiguous angular sector. The number of target detections and ambiguous localization results provided by each DVB-T channels are illustrated in Table I. As illustrated in Section III, the joint exploitation of multiple frequencies allows to broaden the unambiguous angular sector.

This is clearly apparent by observing Fig. 5, where a centralized detection scheme using all the three available channels is adopted [6], while the proposed MF ML approach is considered for DoA estimation. Specifically, the maximization of (5) is performed over a wide angular sector

TABLE I. PERFORMANCE COMPARISON BETWEEN SF AND MF DOA ESTIMATION APPROACHES, WHEN TWO SURVEILLANCE ANTENNAS ARE EMPLOYED.

\begin{tabular}{|c|c|c|}
\cline { 2 - 3 } \multicolumn{1}{c|}{} & Detections & $\begin{array}{c}\text { Ambiguous } \\
\text { plots }\end{array}$ \\
\hline Single DVB-T channel $f_{1}$ & 26 & 7 \\
\hline Single DVB-T channel $f_{2}$ & 30 & 14 \\
\hline Single DVB-T channelf $f_{3}$ & 26 & 12 \\
\hline $\begin{array}{c}\text { Multiple DVB-T channels } \\
\boldsymbol{f}_{\mathbf{1}}, \boldsymbol{f}_{\mathbf{2}}, \boldsymbol{f}_{3}\end{array}$ & $\mathbf{3 3}$ & $\mathbf{2}$ \\
\hline
\end{tabular}

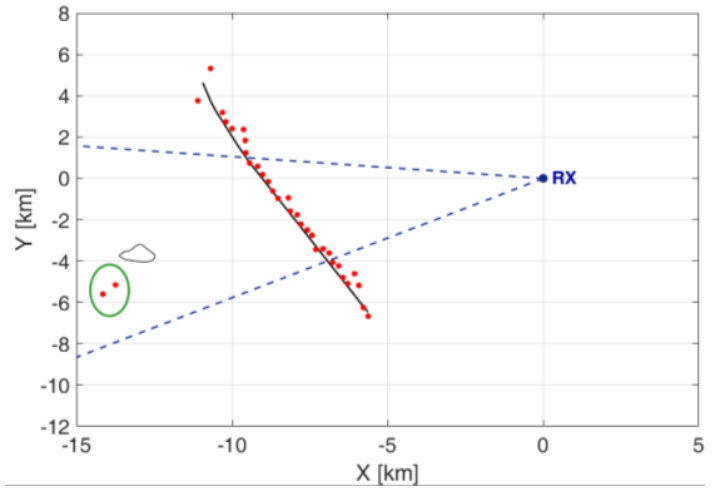

Fig. 5. Localization results for 34 consecutive data files using two surveillance antennas and exploiting all three DVB-T channels.

equal to $\left[-80^{\circ}: 0.01^{\circ}: 80^{\circ}\right]$. As is apparent, the MF solution allows to avoid the ambiguous estimation of the DoA for targets' plots outside the SF angular sector. However, there is a non-zero probability that outliers could occur when two antennas are considered. As illustrated in Table I, two ambiguous localizations are obtained, also highlighted with green circles Fig. 5.

As aforementioned (see Section III), an alternative way to widen the unambiguous angular sector is the use of NULA. Fig. 6(a) sketches the results obtained when using three surveillance antennas and exploiting the single DVB-T channel $f_{2}$. By comparing Fig.4 (a) and Fig. 6 (a), the amount of ambiguous plots turns from 14 to just 1 . Table II reports both the number of detections and ambiguously localized plots for all the considered DVB-T channels, for the case of three surveillance antennas. By comparing Table I and Table II, we might notice that a significantly reduced number of ambiguous plots is obtained when exploiting three receiving antennas for all the SF operations.

Eventually, we considered the case of using three nununiformly spaced surveillance antennas and the three available DVB-T channels. The localization results for this case are sketched in Fig. 6 (b), where we notice that the total amount of detected target plots is unambiguously localized in the XY plane.

Moreover, it is worth mentioning that a further improvement is offered by the proposed strategy in terms of DoA estimation accuracy. In fact, the advantage is evident if we compare the proposed solution (see Fig. 6(b)) with the exploitation of two receiving antennas, when the best DVB-T channel is employed (see Fig.4). This results is confirmed also by observing Fig. 7, where the angular errors are reported as a function of the acquisition time for three configurations: two and three surveillance antennas using the best DVB-T channel, in blue and black, respectively and three surveillance antennas using the MF approach, in green. Similar conclusions can be drawn by comparing the proposed solution (see Fig. 6(b)) with the exploitation of two receiving antennas and multiple DVB-T (see Fig. 5).

Additionally, as expected, when three surveillance antennas are used, only a slight improvement is obtained with the MF with respect to the SF approach. It is worth noticing that the latter improvement does not appear substantial since we are comparing the MF approach with the best single DVB$\mathrm{T}$ channel, while the remaining two frequency channels included in the non-coherent integration do not bring a beneficial contribution in terms of both likelihood function 


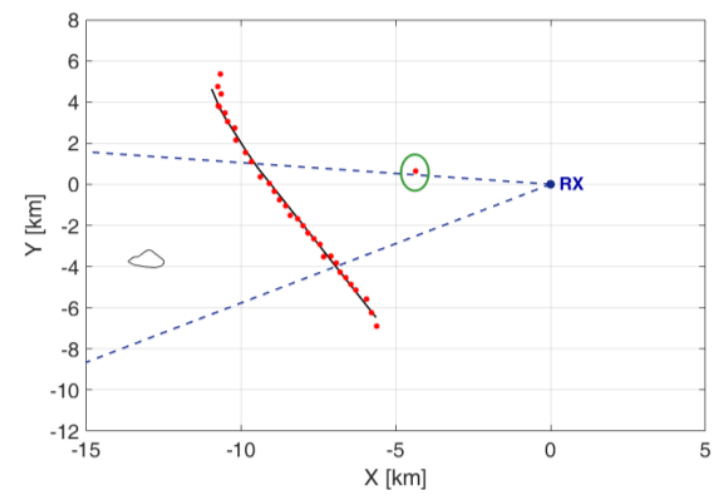

(a)

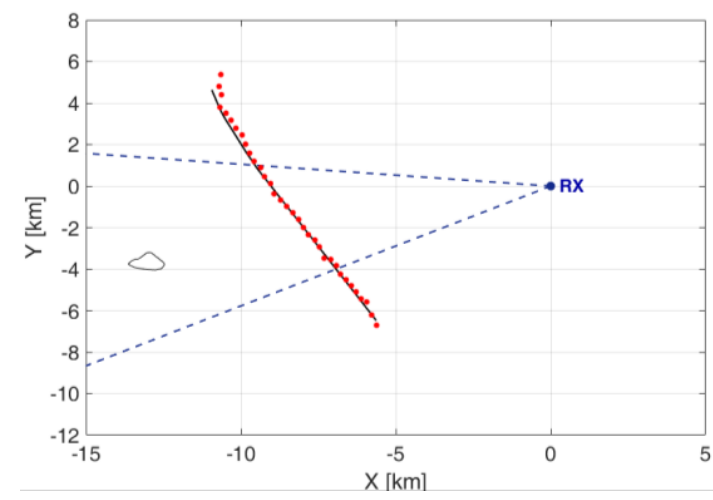

(b)

Fig. 6. Localization results for 34 consecutive data files using three surveillance antennas and exploiting:

(a) the best single DVB-T channel $\left[f_{2}\right]$; (b) the MF approach with the joint exploitation of all three DVB-T channels.

shape and equivalent SNR. An extensive analysis of the DoA estimation accuracy over several targets will be considered for future activities.

\section{CONCLUSIONS}

In this paper, we address the problem of the target DoA estimation in passive radar systems exploiting DVB-T signals as sources of opportunity. In order to relax the typical tradeoff between DoA estimation accuracy and unambiguous angular sector, we resort to a non-uniform arrangement of the surveillance antenna elements and we jointly exploit the signals simultaneously collected at different carrier frequencies. The application against experimental data sets provided by Leonardo S.p.A, allowed to illustrate the benefits of the proposed approach. Specifically we have shown that, even operating with few antenna elements arranged in a given layout, the exploitation of frequency diversity allows to:

(i) extend the angular sector where the target DoA can be unambiguously estimated thanks to the wide frequency diversity provided by the exploitation of DVB-T signals

TABLE II. PERFORMANCE COMPARISON BETWEEN SF AND MF DOA ESTIMATION APPROACHES, WHEN THREE SURVEILLANCE ANTENNAS ARE EMPLOYED

\begin{tabular}{|c|c|c|}
\cline { 2 - 3 } \multicolumn{1}{c|}{} & Detections & $\begin{array}{c}\text { Ambiguous } \\
\text { plots }\end{array}$ \\
\hline Single DVB-T channel $f_{1}$ & 28 & 2 \\
\hline Single DVB-T channel $f_{2}$ & 32 & 1 \\
\hline Single DVB-T channelf $f_{3}$ & 26 & 2 \\
\hline $\begin{array}{c}\text { Multiple DVB-T channels } \\
\boldsymbol{f}_{\mathbf{1}}, \boldsymbol{f}_{\mathbf{2}}, \boldsymbol{f}_{\mathbf{3}}\end{array}$ & $\mathbf{3 4}$ & $\mathbf{0}$ \\
\hline
\end{tabular}

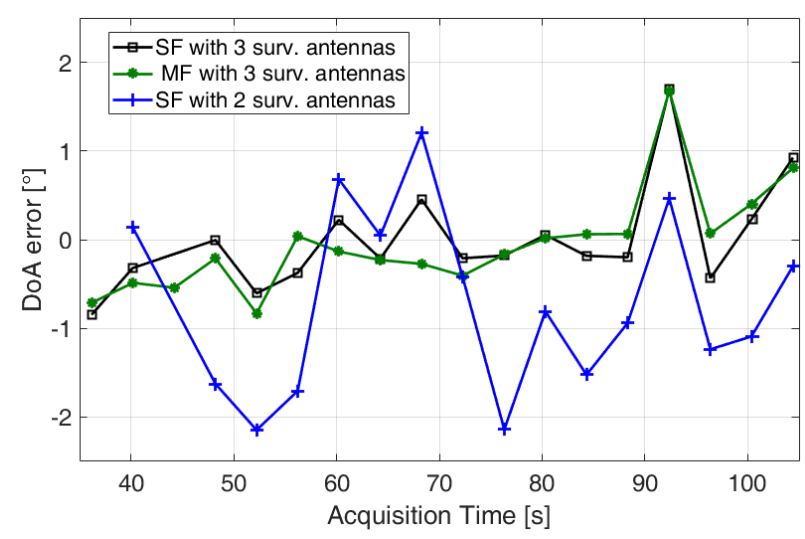

Fig. 7. DoA estimation accuracy for the considered target of opportunity, with

- a single DVB-T channel $\left[f_{2}\right]$ with two (blue) or three (black) surveillance antennas

- three DVB-T channels with three surveillance antennas (green)

(ii) improve the target DoA estimation accuracy thanks to the enhancement of target equivalent SNR resulting from the integration across the frequency channels.

It is worth noticing that, although the presence of some ambiguous plots might represent a minor problem when the corresponding detections have already been included in a tracking stage on the range-velocity plane, they could be an issue in a track initialization phase, especially in more complicated systems, for instance in a multistatic configuration.

\section{REFERENCES}

[1] A.Farina, H.Kuschel: "Special Issue on Passive Radar", IEEE Aerospace and Electronic Systems Magazine, vol. 27, no. 10, 2012.

[2] H.L.Van Trees: "Optimum Array Processing: Part IV of Detection, Estimation, and Modulation Theory", John Wiley \& Sons, Inc., New York, USA (2002)

[3] F.Athley: "Threshold region performance of maximum likelihood direction of arrival estimators", IEEE Transactions on Signal Processing, vol. 53, no. 4, pp. 1359-1373, April 2005.

[4] H.Sun, L.Wan, X.Lan, L.Xie: "Target DOA Estimation Using Nonuniform Sparse Array for Low Frequency Radar", Proceedings of International Conference on Radar Systems, October 2017, in print

[5] F. Colone, C. Bongioanni, P. Lombardo: "Multifrequency integration in FM radio-based passive bistatic radar. Part I: Target detection", IEEE Aerospace and Electronic Systems Magazine, vol.28, no.4, pp.28-39, April 2013.

[6] T.Martelli, F.Colone, E.Tilli, A.Di Lallo: "Multi-Frequency Target Detection Techniques for DVB-T Based Passive Radar Sensors", Sensors, vol. 16, pp. 1594, 2016.

[7] K.E.Olsen, K.Woodbridge: "Performance of a multiband passive bistatic radar processing scheme - Part I", IEEE Aerospace and Electronic Systems Magazine, vol. 27, pp. 16-25, 2012

[8] K. E.Olsen, K. Woodbridge: "Multiband passive bistatic DVB-T radar range resolution improvements and implications", Proceedings of 13th International Radar Symposium, pp. 28-31, May 2012.

[9] D.Olivadese, E.Giusti, D.Petri, M.Martorella, A.Capria, F.Berizzi: "Passive ISAR with DVB-T signals", IEEE Transactions on Geoscience and Remote Sensing, vol. 51, no. 8, pp. 4508-4517, August 2013.

[10] F.Colone, C.Bongioanni, P. Lombardo: "Multifrequency integration in FM radio-based passive bistatic radar. Part II: Direction of arrival estimation" IEEE Aerospace and Electronic Systems Magazine, vol.28, no.4, pp.40-47, April 2013.

[11] F.Colone, D.W. O'Hagan, P.Lombardo, C.J.Baker: "A multistage processing algorithm for disturbance removal and target detection in passive bistatic radar", IEEE Transactions on Aerospace and Electronic Systems, vol. 45, no. 2, Apr. 2009 